\title{
ADVANCED FUEL CELL SYSTEM CONTROL THROUGH STATE SPACE MODELING
}

\author{
Felix Grasser ${ }^{* 1}$ Alfred Rufer* \\ * Ecole Polytechnique Fédérale de Lausanne, Laboratoire \\ d'Electronique Industrielle, CH-1015 Lausanne, \\ Switzerland
}

\begin{abstract}
Maintaining a fuel cell system in correct operating conditions when subjected to fast load changes requires good system control. In order to design such a controller, a simple PEFC system model describing all the important system dynamics and cross couplings is required.

The simple model shown here describes the fuel cell auxiliary systems in a state space approach, linking user input to fuel cell operating conditions. A steady state fuel cell stack model then describes the effect of those operating conditions on system performance.

The model then allows implementation of advanced control strategies such as multivariable control with decoupling, actuator sensitivity analysis or maximum efficiency tracking algorithms.
\end{abstract}

Keywords: Fuel Cell, Model, State Space, Control System

\section{INTRODUCTION}

If fuel cell (FC) technology is to be used as decentralized power sources or in mobile or transportation applications, the systems have to be able to adapt to fast load changes and varying operating parameters. In order to achieve such performance, the system's behaviour has to be understood and appropriately modelled. This knowledge can then be leveraged to design advanced control strategies that allow running the system at maximum efficiency while maintaining it in a healthy operating regime.

While full featured 3D or 2D finite element models can accurately describe the complicated phenomena occurring in the FC, their numerical nature makes them unsuitable for use in an real-

\footnotetext{
1 Financial support by the Swiss Federal Office of Energy is gratefully acknowledged.
}

time system controller. A simple, computationally non-intensive (ideally fully analytical) FC system model describing the dominant system time constants is therefore required.

In order to achieve these goals, a typical Polymer Electrolyte Fuel Cell (PEFC) System will be considered. In a first step, the system's auxiliaries responsible for providing reaction gas flow and conditioning along with temperature control will be described in a dynamic, state-space inspired approach. Those models link the user (or ultimately controller) input to the resulting effect on the PEFC System. They are shown as "Air", " $\mathrm{H}_{2}$ " and "Thermal" subsystems in Figure 1.

The next step consists of describing the effect of the operating conditions (imposed through the auxiliary systems) on the FC stack. In order to achieve this, species transport is described in an averaged 1D reference frame. The resulting 


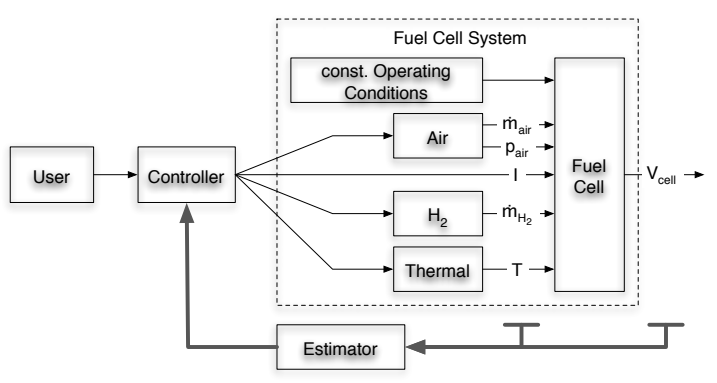

Figure 1. Schematic representation of the system and subsystem models.

reactant concentration at the electrode interface along with the water content of the membrane allows to determine the overall stack voltage.

The resulting state space models for the system's auxiliaries along with the stack model can then be combined into an overall system state space model that will serve as the basis for implementing advanced control strategies. Currently, in most fuel cell applications, SISO controllers are used for the different subsystems with no way of accounting for the cross-couplings in the overall system. The state space model allows to account for these effects through multivariable control and estimation of non-measurable quantities. Typically, this would be used for automatic control of water management or overall system efficiency - both areas where currently appropriate operating conditions are chosen a priori from which the system cannot deviate and therefore only performs optimally on that one operating point.

Note that because of the limited scope of this article, the complex problems of water management will only be outlined but not included in the model presented here.

\section{SYSTEM DESCRIPTION AND MODEL}

The FC system being considered in this article is shown in Figure 2. It is composed of an air and hydrogen supply and conditioning system, a cooling circuit along with the fuel cell stack. The following paragraphs give a brief overview of the functions assumed by the different subsystems.

A volumetric air compressor feeds air into the cathode manifold where it passes through a humidification system and enters the FC stack at a given temperature and relative humidity. From the cathode, where part of the oxygen is consumed by the electrochemical reaction, it exits the FC through a water separator into ambient air.

On the hydrogen side, the fuel is introduced into the anode manifold and from there into the FC through a pressure regulator. Upon exiting the FC stack, the leftover hydrogen is re-injected into the anode manifold by a volumetric hydrogen pump.

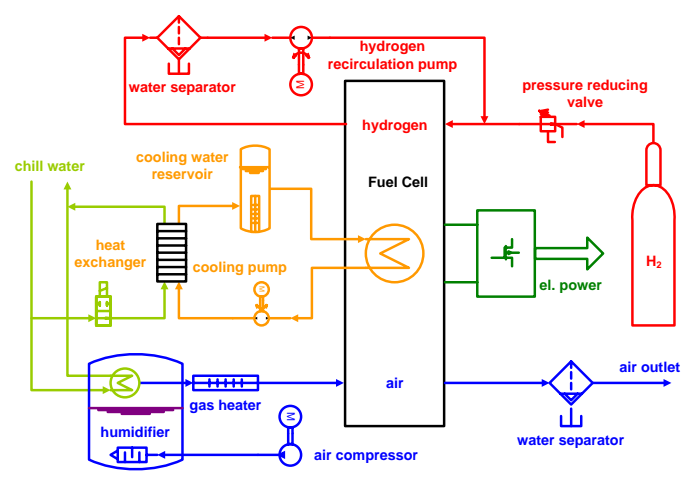

Figure 2. Schematic representation of a PEMFC system

The generated electricity is either fed into a given load or injected into the grid through an adapted power convertor. Heat is rejected through a water cooling circuit connected to a liquid/liquid heat exchanger. Cooling water flow is provided by a small, speed controllable water pump.

\subsection{Air Supply Subsystem Model}

The air supply subsystem consisting of an air compressor (CO), a humidification system, the fuel cell cathode compartment along with the water separator at the exit is shown in Figure 2. From a controls point of view, the user input to the air supply subsystem is the voltage applied to the compressor - the resulting outputs are the air flowrate and pressure in the FC cathode compartment.

For the air supply dynamics, the humidifier only contributes to the inlet volume and the influence of the water separator at the exit can be neglected. The volume of the FC cathode gas distribution channels is very small compared to the inlet chamber volume $V_{a d m}$ (pipes and humidifier) and can therefore be modelled as a simple airflow resistance. The schematic resulting from these assumptions is shown in Figure 3.

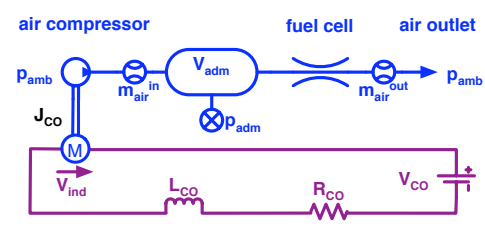

Figure 3. Model schematic for the air supply subsystem

Within such a representation, we can distinguish three different time constants in the system an electrical time constant related to the current in the compressor, a mechanical time constant related to the inertia of the compressor and a hydraulic time constant related to the volume in the air supply system. 
The governing equation for the electrical part relates the compressor voltage $V_{C O}$ to the compressor current $i_{C O}$ and speed $\omega_{C O}$.

$$
V_{C O}=L_{C O} \frac{d i_{C O}}{d t}+R_{C O} \cdot i_{C O}+k_{t} \cdot \omega_{C O}
$$

$L_{C O}$ is the motor inductance, $R_{C O}$ the electrical motor resistance and $k_{t}$ the torque constant. The mechanical compressor speed can be shown to be modelled by the following equation if we assume a load torque proportional to the pressure difference between inlet and outlet, a compressor inertia $J_{C O}$ and a friction torque proportional to compressor speed.

$$
\begin{aligned}
J_{C O} \cdot \dot{\omega}_{C O}= & k_{t} \cdot i_{C O}-k_{f r i c} \cdot \omega_{C O} \\
& -k_{p}\left(p_{a d m}-p_{a m b}\right)
\end{aligned}
$$

$k_{p}$ is the compressor's pressure-torque coefficient, $k_{\text {fric }}$ is the friction coefficient and $p_{a d m}$ and $p_{a m b}$ the FC inlet manifold and ambient pressure respectively.

Assuming constant temperature and ideal gas law behaviour, the term describing the pressure dynamics is expressed by (3).

$$
\frac{d p_{a d m}}{d t}=\frac{\mathcal{R} T}{V_{a d m} \mathcal{M}_{a i r}}\left(\dot{m}_{a i r, \text { in }}-\dot{m}_{a i r, o u t}\right)
$$

$\mathcal{M}_{\text {air }}$ is the molar air mass, $\mathcal{R}$ the universal gas constant, $T$ the air temperature, $V_{a d m}$ the inlet manifold volume and $\dot{m}_{a i r, i n}$ and $\dot{m}_{a i r, o u t}$ the inlet and outlet massflowrate respectively. The outlet air flowrate can be described as a function of the pressure drop across the fuel cell stack. For a laminar flowregime typical of fuel cell applications, it can be shown that the pressure drop is linear with the air flowrate (i.e. $\dot{m}_{a i r, o u t}=k_{h}\left(p_{a d m}-p_{a m b}\right)$ where $k_{h}$ is the hydraulic air flow resistance of the FC). Additionally, for a volumetric compressor, the resulting flowrate is proportional to the compressor speed. (i.e. $\dot{m}_{a i r, i n}=k_{m} \cdot \omega_{C O}$ where $k_{m}$ is the compressor's massflow-speed constant).

Equations (1), (2) and (3) can then be combined in matrix notation to give a state space description of the air supply subsystem.

$$
\begin{aligned}
{\left[\begin{array}{l}
\frac{d i_{C O}}{d t} \\
\frac{d \omega_{C O}}{d t} \\
\frac{d p_{a d m}}{d t}
\end{array}\right]=} & {\left[\begin{array}{ccc}
A_{11} & A_{12} & 0 \\
A_{21} & A_{22} & A_{23} \\
0 & A_{32} & A_{33}
\end{array}\right]\left[\begin{array}{c}
i_{C O} \\
\omega_{C O} \\
p_{a d m}
\end{array}\right] } \\
& +\left[\begin{array}{cc}
B_{11} & 0 \\
0 & B_{23} \\
0 & B_{32}
\end{array}\right]\left[\begin{array}{c}
V_{C O} \\
p_{a m b}
\end{array}\right]
\end{aligned}
$$

$$
\left[\begin{array}{c}
\dot{m}_{a i r} \\
p_{a d m}
\end{array}\right]=\left[\begin{array}{ccc}
0 & k_{m} & 0 \\
0 & 0 & 1
\end{array}\right]\left[\begin{array}{c}
i_{C O} \\
\omega_{C O} \\
p_{a d m}
\end{array}\right]
$$

In this context, the humidifier is not included in the model. Inlet air humidity is accounted for through the measurement of the air dew point in the inlet manifold.

\subsection{Hydrogen Supply Subsystem Model}

The hydrogen supply subsystem is composed of a pressure reducing valve connecting it to the hydrogen source, a hydrogen recirculation pump and the FC anode compartement (see Figure 2). From a controls point of view, the user input to the hydrogen supply subsystem is the voltage applied to the pump - the resulting output is the hydrogen flowrate through the FC anode compartment.

The inlet hydrogen pressure is directly controlled by the closed-loop (mechanically) pressure regulation valve that imposes the dynamics. Additionally, the hydrogen inlet volume is much smaller such that we can neglect any pressure variation dynamics on the hydrogen side. An approach similar to the one taken with the air supply subsystem then results in a $2 \times 2$ state space model describing the dynamics of the hydrogen massflow and pump current.

\subsection{Thermal Management Subsystem Model}

The thermal management subsystem is composed of the cooling channels integrated into the fuel cell, a variable speed cooling liquid (CL) pump and a liquid-liquid heat exchanger (HX) to reject heat to a chill water $(\mathrm{CW})$ circuit as shown in Figure 2. The experimental setup also integrates a cooling water reservoir (RV) with an electrical heating element, allowing the system to be brought to operating temperature prior to switch on along with maintaining operating temperature in low load conditions.

Because of the slow thermal time constants, the cooling pump is simply described through a steady state model providing the cooling liquid flowrate $\dot{m}_{C L}$ as a function of the applied voltage $V_{C L}$.

The FC temperature $T_{F C}$ is assumed uniform. Applying a basic energy balance for the fuel cell plates then allows to express the evolution of the plate temperature as a first order differential equation. A similar energy balance approach expresses the cooling liquid reservoir's temperature evolution. The Heat Exchanger is considered a heat transfer element only. Performance is described as 
a function of the heat exchanger efficiency along with the chill water flowrate.

The resulting equations can then be regrouped and linearized to form a state space expression of the of the system with states $\mathbf{x}=\left[T_{F C} T_{R V}\right]^{T}$ and input $\mathbf{u}=\left[\begin{array}{llll}\dot{Q}_{\text {loss }} & \dot{Q}_{\text {heat }} \dot{m}_{C L} T_{C W} & d\end{array}\right]^{T}$. $\dot{Q}_{\text {loss }}$ represents the fuel cell losses and is calculated from the FC performance equation (7), $\dot{Q}_{\text {heat }}$ is the heating power provided by the external heating cartridge located in the water reservoir, $\dot{m}_{C L}$ is the cooling liquid flowrate, $T_{C W}$ the chill water temperature and $d$ the duty cycle of the valve providing chill water flow to the heat exchanger.

\section{FUEL CELL STACK DESCRIPTION AND MODEL}

As mentioned in section 1, the goal is to describe the overall FC voltage based on the operating conditions. From a controls point of view, only the fuel cell current $I_{F C}$ is a direct user input to the $\mathrm{FC}$ - all other operating conditions being the result of the user's (or the control system's) interaction with the auxiliary systems (see Figure 1). This can be written as a general equation in the form of

$$
\begin{aligned}
V_{F C}= & f_{1}\left(\dot{m}_{a i r}, p_{a i r}, \dot{m}_{H_{2}}, T_{F C}\right)+f_{2}\left(I_{F C}\right) \\
& +f_{3}(\text { const. operating conditions })
\end{aligned}
$$

Section 3.1 will briefly explain the mechanisms influencing fuel cell performance - the different equations composing $f_{1}$ to $f_{3}$ in (6) (but not yet as a direct function of the operating conditions) will be given.

Section 3.2 then focuses on how to tie the performance equations to the operating conditions and system inputs by describing species transport to and from the reaction sites.

Currently, this model describing gas transport to the reaction sites along with water transport across the cell represents steady state behaviour. While this is fine for relatively fast gas transport phenomena, it is not correct for water transport where the polymer membrane acts like a storage element and introduces slow dynamic behaviour.

\subsection{Fuel Cell Performance Description}

The overall stack voltage is a sum of many different contributions related to various physical phenomena. Typically it is expressed as a certain equilibrium voltage - termed the Nernst voltage $E_{0}$ (see p.ex. (Kordesch and Simader, 1996)) and dependent on temperature and reactant partial pressures - from which losses are deducted. The overall cell voltage is then written as:

$$
V_{F C}=E_{0}-\eta_{a c t}-\eta_{c o n c}-\eta_{o h m}-\eta_{i o n}
$$

The losses typically encountered in FC operation are:

$\eta_{\text {act }}$ - Activation losses describe the resistance to charge transfer at each of the half cells. They are the principal source of losses at low current densities and occur at each electrode of the fuel cell.

An overall description of the overpotential can be made through the exchange current density $I_{0, c}^{\prime \prime}$ typical of the catalyst layer in question. In simplified form, this yields the Tafel equation which gives a direct expression for the activation losses as a function of the fuel cell current density $I_{F C}^{\prime \prime}$ (i.e. area specific current)

$$
\eta_{a c t}=\frac{\mathcal{R} T_{F C}}{\beta n F}\left[\ln \left(I_{0, c}^{\prime \prime}\right)-\ln \left(I_{F C}^{\prime \prime}\right)\right]
$$

where $\beta$ is the reaction symmetry coefficient. Note that (8) expresses the activation overpotential for the cathode only. It can be shown that the activation losses (overpotential) at the anode are very small compared to the ones on the cathode and can generally be neglected (Bernardi and Verbrugge, 1992).

$\eta_{\text {conc }}$ - Concentration losses describe the losses due to gas transport losses in the cell. If no current is drawn, the reactant concentrations at the reaction surface (i.e. catalyst layer - membrane interface) correspond to those in the gas channel. As soon as a certain net current is drawn from the fuel cell, reactants have to diffuse to the catalyst layer at a rate corresponding to the electric current. This diffusion reduces the reactant concentration at the reaction surface.

The concentration overpotential $\eta_{\text {conc }}$ is the difference between the Nernst potential corresponding to the partial reactant pressures in the gas channel, $E_{0, G C}$, and the one corresponding to the partial reactant pressures at the reaction surface, $E_{0, S}$. It is written as a function of the partial reactant pressures in the gas channel $p_{i, G C}$ and at the reaction surface $p_{i, S} \cdot \gamma_{i}$ is the stochiometric factor of component $i$ in the reaction where the subscript $i$ refers to an educt and $j$ to a product.

$$
\begin{aligned}
\eta_{\text {conc }} & =E_{0, G C}-E_{0, S} \\
& =-\frac{\mathcal{R} T_{F C}}{n F} \ln \left(\frac{\prod_{i} p_{i, G C}^{\gamma_{i}}}{\prod_{j} p_{j, G C}^{\gamma_{j}}} \frac{\prod_{j} p_{j, S}^{\gamma_{j}}}{\prod_{i} p_{i, S}^{\gamma_{i}}}\right)
\end{aligned}
$$

$\eta_{\mathbf{o h m}}$ - Electronic conduction losses describe the losses due to electron conduction in the gas diffusion layer (GDL) and the bipolar plates 
(BP). $R_{G D L}$ and $R_{B P}$ can be regrouped in an overall equivalent FC resistance $R_{e q}$.

$$
\eta_{o h m}=\left(R_{G D L}+R_{B P}\right) I_{F C}=R_{e q} I_{F C}
$$

$\eta_{\text {ion }}$ - Ionic losses describe the losses due to proton transport through the polymer membrane. Like the electronic conduction losses, they can be described as an ohmic overpotential. However, in the case of the ionic overpotential, the membrane conductivity is strongly dependent on the membrane water content $\xi$ which has been widely shown in literature (Rieke and Vanderborgh, 1987). The expression proposed by Springer et al. in (Springer et al., 1991) was adopted to describe the membrane conductivity $\sigma_{m}=f(\xi)$ as a function of the membrane water content $\xi$. The ionic overpotential can then be written as a function of the membrane thickness $\delta_{m}$ and the current density $I_{F C}^{\prime \prime}$.

$$
\eta_{i o n}=\frac{I_{F C}^{\prime \prime} \delta_{m}}{\sigma_{m}(\xi)}
$$

The equilibrium cell voltage $E_{0}$, the activation overpotential $\eta_{a c t}(8)$ and the ohmic overpotential $\eta_{\text {ohm }}$ (10) can directly be determined from the operating parameters.

This is not the case for the concentration overpotential $\eta_{\text {conc }}$ (9) and the ionic overpotential $\eta_{i o n}(11)$ which depend on the partial reactant pressures at the reaction interface along with the membrane water content. These quantities are related to species transport within the fuel cell and need to be described appropriately based on the FC operating parameters. The following paragraphs outline a simplified, space averaged model to achieve this.

\subsection{Reaction Gas Transport}

Reaction Gas Transport (i.e. oxygen on the cathode side and hydrogen on the anode side) occurs in two spatial directions:

- pressure difference driven convective transport in the $x-y$ (membrane) plane along the gas channels.

- concentration difference driven diffusive transport in the $z$ direction perpendicular to the membrane. The molar flowrate of the oxygen flow in the $z$-direction is directly related to the fuel cell current through Faraday's Law.

Since the diffusional transport is concentration related, there is a strong dependency on the water transport which directly influences the remaining species concentration. Note that for the remainder of this section, only the cathode gas channel (CGC) will be considered. However, the same reasoning and the same equations also apply to hydrogen transport in the anode gas channel (AGC).

Assuming no gas storage in the gas channel, a mass balance on the different species flowrate (see Figure 4) allows to determine the average concentration of all species in the gas channel $c_{i}^{G C}$.

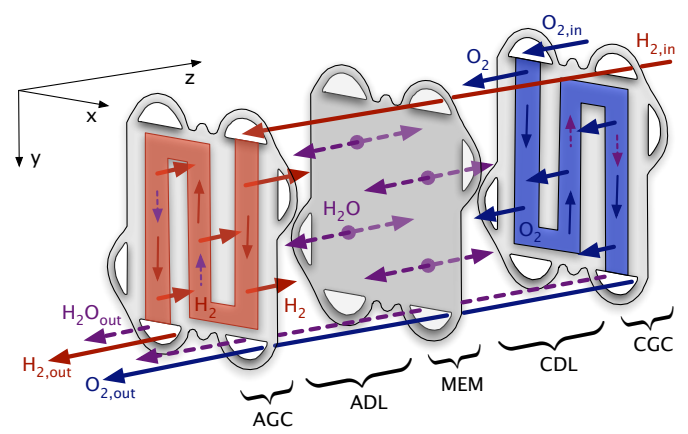

Figure 4. Schematic representation of species transport within a PEMFC repeat element.

Assuming diffusional transport from the gas channel to the catalyst layer, the oxygen concentration at the reaction sites $c_{\mathrm{O}_{2}}^{S}$ (and therefore also its partial pressure at the reaction site) can be expressed as a function of the oxygen flux (in $\frac{m o l}{s \cdot m^{2}}$ ) through the cathode diffusion layer (CDL) $\dot{N}_{O_{2}}^{\prime \prime}$ and the CDL thickness $\delta_{G D L}$.

$$
c_{\mathrm{O}_{2}}^{S}=\frac{\mathcal{R} T_{F C}}{H_{\mathrm{O}_{2}-H_{2} O}}\left(c_{\mathrm{O}_{2}}^{G C}-\frac{\delta_{G D L}}{D_{e q}^{G D L}} \dot{N}_{\mathrm{O}_{2}}^{\prime \prime}\right)
$$

$D_{e q}^{G D L}$ is the equivalent overall GDL diffusivity. The Henry constant $H_{\mathrm{O}_{2}-\mathrm{H}_{2} \mathrm{O}}$ in front of the brackets describes the reduction in oxygen concentration as the oxygen has to dissolve into the water-ionomer mixture surrounding the reaction sites in the catalyst layer.

\subsection{Water Transport}

Water has the particularity of being the only species that can migrate throughout the fuel cell (i.e. from anode to cathode and back). This makes the description of water transport within the cell tricky since transport equations have to be established for all regions within the fuel cell.

The overall water transfer is determined based on

(1) A mass balance consideration on the anode and cathode gas channel respectively yielding the average water concentration in the gas channels.

(2) The water transport equations in the membrane region. Water transport in the membrane can be related to three distinct transport phenomena: Water transport by electro- 
osmotic drag, water transport by diffusion and water transport by convection. More details on the corresponding governing equations can be found in (Springer et al., 1991), (Bernardi and Verbrugge, 1992), and (Rowe and $\mathrm{Li}, 2001$ ).

(3) The water transport equations in the gas diffusion layer (GDL) region.

Solving the above system of governing equations yields the average membrane water content and thus the ionic overpotential (11).

\section{EXPERIMENTAL RESULTS}

The models proposed have been experimentally verified on a test bench composed of the different subsystems along with a 400W PEMFC developed at the Paul Scherrer Institute (Büchi and Ruge, 2001).

As an example illustrating the models for the auxiliary systems, Figure 5 shows the experimental air massflow rate against the modelled flowrate.

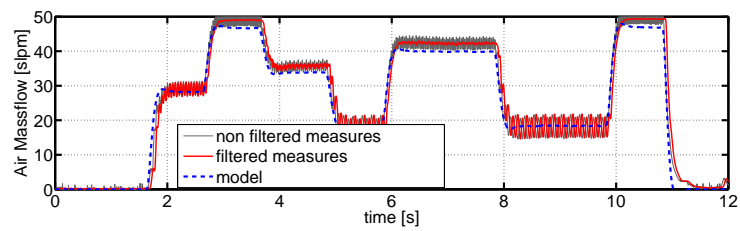

Figure 5. Modelled vs. experimental air massflow in the cathode compartement

Note the fluctuation of the measured flowrate that results from the oscillating movement of the membrane compressor. The model neglects this aspect and only models the average flowrate with a prediction error between $5-10 \%$. Note that the parameters used in the model for Figure 5 have been identified based on separate measurement sets from the ones shown.

Figure 6 depicts an IV curve for the experimental setup (dotted line with o-markers). Operating conditions were $T_{F C}=75^{\circ} C, \quad \lambda_{a}=3, \quad \lambda_{c}=5$, $T_{D P, a i r}=70^{\circ} \mathrm{C}$ where $T_{D P}$ denotes the air dew point and corresponds here to roughly $80 \%$ relative humidity at the FC inlet.

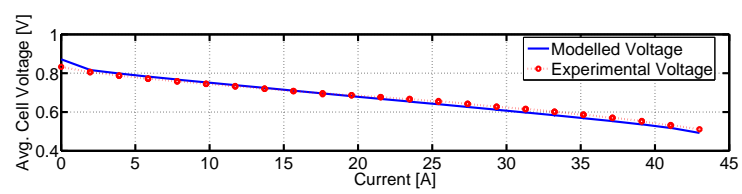

Figure 6. Modelled fuel cell voltage against experimental data

The corresponding modelled voltage is shown as a solid line where a different set of experimental results was used to fit the model parameters. The water transfer coefficient was experimentally identified for the given operating conditions and presumed known during the run of the model.

\section{CONCLUSION}

The model developed has been shown to correctly reproduce the dynamics of the auxiliary systems. The steady state fuel cell stack model can be successfully fitted for a given set of operating conditions.

Note that as mentioned before, the modelling approach for the FC neglects any dynamics in the water transport equations. This does not correspond to what is observed on real cells where a change in humidity levels clearly leads to a dynamic change in the operating point. Further work is necessary to address these shortcomings of the model.

The model can be used to implement a multivariable state space system controller along with an estimator to access system parameters (typically membrane water content) that cannot be measured directly. Further algorithms such as maximum efficiency point tracking (MEPT) can be added based on the system model to further enhance the system efficiency and automatically maintain it in healthy operating conditions.

\section{REFERENCES}

Bernardi, D.M. and M.W. Verbrugge (1992). A mathematical model of the solid-polymerelectrolyte fuel cell. J. Electrochem. Soc. 139(9), 2477 - 2491.

Büchi, F.N. and M. Ruge (2001). Development of a bipolar element for PE fuel cells demonstration in a $6 \mathrm{~kW}$ stack. PSI Scientific Report 2000 (General Energy) V, 84-85.

Kordesch, K. and G. Simader (1996). Fuel Cells and Their Applications. VCH Publishers, Inc.. New York.

Rieke, P.C. and N.E. Vanderborgh (1987). Temperature dependence of water content and proton conductivity in polyperfluorosulfonic acid membranes. Journal of Membrane Science 32, 313 - 328.

Rowe, A. and X. Li (2001). Mathematical modeling of proton exchange membrane fuel cells. Journal of Power Sources 102, 82 - 96.

Springer, T.E., T.A. Zawodzinski and S. Gottesfeld (1991). Polymer electrolyte fuel cell model. J. Electrochem. Soc. 138(8), 2334 2342 . 